\title{
Sub-optimal polynomial meshes on planar Lipschitz domains
}

\author{
F. Piazzon and M. Vianello ${ }^{1}$ \\ Dept. of Mathematics, University of Padova (Italy)
}

November 28, 2013

\begin{abstract}
We construct norming meshes with cardinality $\mathcal{O}\left(n^{s}\right), s=3$, for polynomials of total degree at most $n$, on the closure of bounded planar Lipschitz domains. Such cardinality is intermediate between optimality $(s=2)$, recently obtained by Kroó on multidimensional $C^{2}$ starlike domains, and that arising from a general construction on Markov compact sets due to Calvi and Levenberg $(s=4)$.
\end{abstract}

2000 AMS subject classification: 41A10, 41A63, 65D05.

Keywords: Polynomial Inequalities, Norming Sets, Polynomial Admissible Meshes, Planar Lipschitz Domains.

\section{Introduction}

Polynomial admissible meshes (or polynomial meshes for short), introduced and studied in the seminal paper [7], are sequences $\left\{\mathcal{A}_{n}\right\}$ of norming sets (in the uniform norm) on a multidimensional polynomial determining compact $K \subset \mathbb{R}^{d}$ or $K \subset \mathbb{C}^{d}$ (a polynomial vanishing there vanishes everywhere), i.e., the following polynomial inequality holds

$$
\|p\|_{K} \leq C\|p\|_{\mathcal{A}_{n}}, \quad \forall p \in \mathbb{P}_{n}^{d}
$$

with a cardinality increasing at most like $\mathcal{O}\left(n^{s}\right), s \geq d$; here and below, $\mathbb{P}_{n}^{d}$ denotes the space of $d$-variate polynomials of degree at most $n$, and $\|f\|_{X}$ denotes the sup-norm of a function $f$ bounded on the set $X$. Among their properties, we recall that admissible meshes are preserved by affine

\footnotetext{
${ }^{*}$ Supported by the ex-60\% funds and the biennial project CPDA124755 of the University of Padova, and by the INdAM GNCS.

${ }^{1}$ corresponding author: e-mail: marcov@math.unipd.it
} 
transformations, and can be easily extended by finite union and product [7]. In the present note, we restrict our attention to the real case, i.e., real polynomials and $K \subset \mathbb{R}^{d}$.

Polynomial meshes provide a "good discrete model" of a compact set for many practical purposes. For example, they are nearly optimal for uniform least-squares approximation [7, Thm.1], and contain Fekete-like interpolation subsets with the same asymptotic behavior of the continuous Fekete points of $K$, that can be computed by numerical linear algebra techniques (cf., e.g., [3, 4]). Such approximate Fekete points have been used within spectral element and collocation methods for PDEs (cf. [12, 17]). For a recent and deep survey on polynomial approximation and interpolation in several variables, we refer the reader to [2].

In [7, Thm.5], it has been shown that any (real) compact set which satisfies a Markov polynomial inequality with exponent $r$

$$
\|\nabla p(\boldsymbol{x})\|_{2} \leq M n^{r}\|p\|_{K}, \quad \forall \boldsymbol{x} \in K, p \in \mathbb{P}_{n}^{d},
$$

often called a Markov compact, has a polynomial mesh with $\mathcal{O}\left(n^{r d}\right)$ cardinality (for example, $r=2$ for compact sets which satisfy a uniform interior cone condition).

On the other hand, in the applications it is important to control the cardinality of such discrete models. Indeed, some attention has been devoted to the construction of optimal and near optimal polynomial meshes, which have cardinality $\mathcal{O}\left(n^{d}\right)$ and $\mathcal{O}\left((n \log n)^{d}\right)$, respectively, in compact sets with special geometries (observe that in (1) necessarily $\operatorname{card}\left(\mathcal{A}_{n}\right) \geq \operatorname{dim}\left(\mathbb{P}_{n}^{d}\right) \sim$ $n^{d} / d$ !); cf., e.g., $[6,11,13,15]$. Moreover, the polynomial inequality (1) can be relaxed, asking that it holds with $C=C_{n}$, a sequence of constants increasing at most polynomially with $n$ : in such a case, we speak of weakly admissible polynomial meshes. Weakly admissible meshes with $\mathcal{O}\left(n^{d}\right)$ cardinality and constants $C_{n}=\mathcal{O}\left((\log n)^{d}\right)$ are known in several instances, cf., e.g., $[3,5,10]$.

In the present note we prove constructively that polynomial meshes with $\mathcal{O}\left(n^{3}\right)$ cardinality exist for the closure of planar Lipschitz domains. The cardinality of such meshes is sub-optimal, being intermediate between optimality $(s=d=2)$ and that arising from the general construction by Calvi and Levenberg on Markov compact sets $(s=2 d=4)$.

\section{Planar Lipschitz domains}

We begin by restricting our attention to a special class of planar Lipschitz domains.

Lemma 1 Let $G$ be a planar compact graph domain

$$
G=\left\{(x, y): a \leq x \leq b, g_{1}(x) \leq y \leq g_{2}(x)\right\}
$$


where $g_{1}(x)<g_{2}(x)$ for every $x \in[a, b]$, and $g_{1}, g_{2} \in \operatorname{Lip}[a, b]$.

Then, $G$ has a norming set given by the union of $\mathcal{O}(n)$ curves and a polynomial mesh with $\mathcal{O}\left(n^{3}\right)$ cardinality which lies on the norming curves.

Proof. First, we show in an elementary way that a Markov polynomial inequality holds, since any point of $G$ belongs to a suitable trapezium (triangle) contained in $G$, with minimal distance between pairs of parallel supporting lines that is bounded from below. Indeed, it is well-known that the closure of any bounded Lipschitz domain in $\mathbb{R}^{d}$ is a Markov compact with exponent $r=2$; cf., e.g., [1].

Let $L_{1}$ and $L_{2}$ be the Lipschitz constants of $g_{1}$ and $g_{2}$, respectively, and let define $L=\max \left\{L_{1}, L_{2}\right\}$. Then, it is not difficult to show that any point $(x, y)$ of the graph domain $G$ belongs to a suitable isosceles trapezium, say $\mathcal{T}_{x} \subseteq G$ (depending only on the $x$-coordinate and possibly degenerating into an isosceles triangle). To construct the trapezium (triangle), one first considers the rhombus with opposite vertices in $\left(x, g_{1}(x)\right)$ and $\left(x, g_{2}(x)\right)$, whose sides lie on the lines through such vertices with slope $\pm L$, and then one intersects the rhombus with the graph domain. The required trapezium (triangle) is the wider of the two ones with common major basis on the rhombus diagonal joining $\left(x, g_{1}(x)\right)$ and $\left(x, g_{2}(x)\right)$.

The trapezium major base has length

$$
\Delta(x)=g_{2}(x)-g_{1}(x) \geq \beta=\min _{x \in[a, b]}\left(g_{2}(x)-g_{1}(x)\right),
$$

and the trapezium height is bounded form below by

$$
\min \{\Delta(x) /(2 L),(b-a) / 2\} \geq \min \{\beta /(2 L),(b-a) / 2\} .
$$

Moreover, the distance of one of the oblique sides from the line parallel to it through the opposite vertex, is

$$
\Delta(x) \cos (\arctan (L))=\Delta(x) / \sqrt{1+L^{2}} \geq \beta / \sqrt{1+L^{2}} .
$$

The minimal distance between parallel supporting lines of the trapezium $\mathcal{T}_{x}$, say $\sigma(x)$, is then bounded from below

$$
\sigma(x) \geq w=\min \left\{\frac{\beta}{\max \left\{2 L, \sqrt{1+L^{2}}\right\}}, \frac{b-a}{2}\right\}>0 .
$$

By a well-known result of Wilhelmsen on the Markov polynomial inequality for convex compact sets [16], we get

$$
\|\nabla p(x, y)\|_{2} \leq \frac{4}{\sigma(x)} n^{2}\|p\|_{\mathcal{T}_{x}} \leq M n^{2}\|p\|_{G}, \quad M=\frac{4}{w},
$$

for every $p \in \mathbb{P}_{n}^{2}$, i.e., $G$ satisfies a Markov polynomial inequality with exponent $r=2$. 
By the arguments of [7, Thm.5], it is not difficult to show that $G$ has a polynomial mesh with $\mathcal{O}\left(n^{4}\right)$ points, which is a subset of a $\mathcal{O}\left(n^{2}\right) \times \mathcal{O}\left(n^{2}\right)$ grid. Indeed, to be a polynomial mesh with constant $C=1 /(1-\lambda)$, it is sufficient that for any point $P \in G$ there exists a point $A$ of the mesh such that

$$
|P-A| \leq \delta_{n}=\delta_{n}(c)=\frac{2 c}{M n^{2}},
$$

where

$$
\lambda=\lambda(c)=4 c e^{2 c}<1,0<c<c^{*},
$$

$c^{*}=0.175 \ldots$ being the solution of the equation $4 t e^{2 t}=1$.

We begin by considering a Cartesian grid, with suitable $\mathcal{O}\left(1 / n^{2}\right)$ spacing in the projections of $G$ on the cartesian axes. Take in both directions a spacing $h_{n}$ smaller than $\delta_{n} / \sqrt{2}$, such that $(b-a) / h_{n}$ is a positive integer $\nu$, namely

$$
h_{n}=h_{n}(c)=\frac{b-a}{\nu}, \nu=\left\lceil\frac{b-a}{\delta_{n} / \sqrt{2}}\right\rceil,
$$

and consider the grid $\left\{\left(\xi_{i}, \eta_{j}\right)\right\}$

$$
\begin{gathered}
\xi_{i}=a+(i-1) h_{n}, \quad i=1, \ldots, \frac{b-a}{h_{n}}+1, \\
\eta_{j}=\min g_{1}+(j-1) h_{n}, \quad j=1, \ldots,\left\lceil\frac{\max g_{2}-\min g_{1}}{h_{n}}\right\rceil+1 .
\end{gathered}
$$

Then, (5) is satisfied by the grid points belonging to $G$.

The compact $G$ is now contained in the union of $\mathcal{O}\left(n^{2}\right)$ vertical strip segments. On each strip, consider the "highest" and the "lowest" rectangle of the grid which instersect $G$, fix a point of $K$ in each, say $(u, v)$ and $(z, w)$ (it is sufficient to choose the points corresponding to the maximum of $g_{2}$ and the minimum of $g_{1}$ in the strip, respectively). Take these two points together with the intersection points of the vertical lines $x=u$ and $x=z$ with the horizontal lines of the grid between $y=w=g_{1}(z)$ and $y=v=g_{2}(u)$ (observe that all such intersection points belong necessarily to the graph domain $G$ ). The points obtained by this construction clearly belong to a new $\mathcal{O}\left(n^{4}\right)$ polynomial mesh for $G$, with constant $C=1 /(1-\lambda)$, since by construction they still satisfy property (5).

It is clear that the union of the segments $\left\{x=x_{i}, g_{1}\left(x_{i}\right) \leq y \leq g_{2}\left(x_{i}\right)\right\}$ is a norming set for $G$, with the same constant $C$. Now, take for example the Chebyshev points of degree $\lceil\mu n\rceil$ on each segment, namely the points

$$
\left\{\left(x_{i}, y_{i k}\right)=\left(x_{i}, g_{2}\left(x_{i}\right)\left(1+\tau_{k}\right) / 2+g_{1}\left(x_{i}\right)\left(1-\tau_{k}\right) / 2\right)\right\}
$$

where the $\left\{\tau_{k}\right\}, 1 \leq k \leq\lceil\mu n\rceil$, are the zeros of $T_{\lceil\mu n\rceil}(t), \mu>1$, in $(-1,1)$. Since these points form an a polynomial mesh for the segment, with constant $1 / \cos (\pi / 2 \mu)$, by a well-known result of Ehlich and Zeller [9], the union of 
the points of all the segments is then a polynomial mesh for $G$, say $\mathcal{A}_{n}$, such that

$$
\|p\|_{G} \leq \frac{1}{(1-\lambda) \cos (\pi / 2 \mu)}\|p\|_{\mathcal{A}_{n}}, \quad \forall p \in \mathbb{P}_{n}^{2},
$$

with $\operatorname{card}\left(\mathcal{A}_{n}\right)=\mathcal{O}\left(n^{2}\right) \times\lceil\mu n\rceil=\mathcal{O}\left(n^{3}\right)$. Observe now that this mesh lies on the union of the $\lceil\mu n\rceil$ curves

$$
\Gamma_{k}=\left\{\left(x, f_{k}(x)\right), x \in[a, b]\right\}, f_{k}(x)=g_{2}(x)\left(1+\tau_{k}\right) / 2+g_{1}(x)\left(1-\tau_{k}\right) / 2,
$$

$1 \leq k \leq\lceil\mu n\rceil$, which thus form a norming set for $G$.

Remark 1 Our result improves that of [11], in the case of planar graph domains with $g_{1}, g_{2} \notin C^{4}$. Indeed, in [11] it is proved that any planar graph domain with $g_{1}, g_{2} \in C^{k}$ possesses a polynomial mesh with $\mathcal{O}\left(n^{2+4 / k}\right)$ points.

Remark 2 It is worth noticing that the fulfillement of the interior cone condition, or even the existence of a Markov polynomial inequality, is not necessary for a graph domain to possess a polynomial mesh. For instance, in Example 3 in [11], a polynomial mesh with cardinality $\mathcal{O}\left(n^{3}\right)$ has been constructed in a cartesian graph domain with an exponential cusp, that does not satisfy a Markov inequality for any exponent.

More generally, in [2] an elementary but smart argument shows that any polynomial determining compact set $K \subset \mathbb{C}^{d}$ admits a polynomial mesh of cardinality $\mathcal{O}\left((n \log n)^{d}\right)$. However, this result is based on the Fekete points of $K$, which are explicitly known in very few intances and are extremely hard to compute.

Remark 3 Continuity of $g_{1}$ and $g_{2}$ suffices for a graph domain to possess a norming set given by the union of $\mathcal{O}(n)$ curves, and this can be proved with a reasoning similar to that developed above. Indeed, consider a cartesian graph domain like (3), and any of the segments $I(z)=\left\{(z, y): g_{1}(z) \leq y \leq\right.$ $\left.g_{2}(z)\right\}$ for a fixed value of $z \in[a, b]$. The points $\mathcal{A}_{n}(z)=\left\{\left(z, g_{2}(z)\left(1+\tau_{k}\right) / 2+\right.\right.$ $\left.\left.g_{1}(z)\left(1-\tau_{k}\right) / 2\right), 1 \leq k \leq\lceil\mu n\rceil\right\}$ form a polynomial mesh for the segment, thus $\bigcup_{z \in[a, b]} \mathcal{A}_{n}(z)$ is a norming set for $G$ with constant $C=1 / \cos (\pi / 2 \mu)$, which concides with the union of the $\lceil\mu n\rceil$ curves (11).

We are now ready to state and prove the main result of this note.

Theorem 1 Let $K=\bar{\Omega} \subset \mathbb{R}^{2}$ be the closure of a bounded Lipschitz domain. Then, $K$ has a polynomial mesh with $\mathcal{O}\left(n^{3}\right)$ cardinality.

Proof. Roughly speaking, Lipschitz domains can be characterized as domains which are locally epigraphs of Lipschitz continuous functions. We refer the reader, e.g., to [8, Ch. 5] for definitions and properties. 
It is not difficult to show by compactness that $\partial \Omega=\partial K$ can be covered by a finite number of (compact) rectangles, say $\mathcal{R}_{i}, i=1, \ldots, m$, such that the intersection of $K$ with each rectangle is a compact graph domain of the form (3) with $g_{1}=0$ (in suitable local Cartesian coordinates, i.e., up to rotation and translation).

This entails that $K \cap \bigcup \mathcal{R}_{i}$ has a polynomial mesh, say $\mathcal{A}_{n}^{1}$, with $\mathcal{O}\left(n^{3}\right)$ cardinality, given by the union of meshes of the corresponding Lipschitz graph domains. Indeed, we recall that a finite union of polynomial meshes is a polynomial mesh for the corresponding union of compact sets, whose constant $C$ is the maximum of the corresponding constants.

On the other hand, $K \backslash \bigcup \mathcal{R}_{i}$ is an open set, whose boundary is contained in $\bigcup \partial \mathcal{R}_{i}$. Hence, its connected components have as boundary a polygonal path, i.e., $K \backslash \bigcup \mathcal{R}_{i}$ is a finite union of open polygons. It is known that any compact polygon has a polynomial mesh with $\mathcal{O}\left(n^{2}\right)$ cardinality, thus by finite union the closure of $K \backslash \bigcup \mathcal{R}_{i}$ has a polynomial mesh, say $\mathcal{A}_{n}^{2}$, with $\mathcal{O}\left(n^{2}\right)$ cardinality. Then, $\mathcal{A}_{n}^{1} \cup \mathcal{A}_{n}^{2}$ is a polynomial mesh for $K$ with $\mathcal{O}\left(n^{3}\right)$ cardinality.

Lipschitz domains can be also characterized as domains that satisfy a uniform interior cone condition $[8, \S 2.6]$, and thus they satisfy a Markov polynomial inequality with exponent $r=2$. The general construction by Calvi and Levenberg in [7, Thm. 5] would then ensure the existence of a polynomial mesh with $\mathcal{O}\left(n^{4}\right)$ cardinality, whereas Theorem 1 reduces the cardinality to $\mathcal{O}\left(n^{3}\right)$.

The result can be extended to dimension $d>2$, by generalizing Lemma 1 to $d$-dimensional Lipschitz graph domains; the construction in the proof of Theorem 1 would involve, in this case, $d$-dimensional polytopes, where existence of polynomial meshes with $\mathcal{O}\left(n^{d}\right)$ cardinality is known [11]. The result, however, becomes less interesting in possible applications, since increasing $d$ the cardinality of the resulting polynomial mesh, $\mathcal{O}\left(n^{2 d-1}\right)$, gets far from $\mathcal{O}\left(n^{d}\right)$ (optimality) and approaches that of the general construction by Calvi and Levenberg, which is $\mathcal{O}\left(n^{2 d}\right)$.

\subsection{Computational issues}

We make now a quantitative comparison of the cardinality of the sub-optimal polynomial mesh as in Lemma 1, with that produced by the general construction of Calvi and Levenberg for Markov compact sets (when specialized to Lipschitz graph domains). We consider for simplicity the case $g_{1} \equiv 0$, $g_{2}=g>0$.

Choose a grid, say $\left\{\left(\xi_{i}, \eta_{j}\right)\right\}$, with spacing $h_{n}$, for a fixed value of $c<c^{*}$, cf. (5)-(8). The construction in [7, Thm. 5] requires the choice of a point of $G$ in each square of the grid which intersects $G$. A simple way to perform this task is to compute the maximum of $g$ in each subinterval of the $x$-grid, 
say $m_{i}=g\left(x_{i}\right), x_{i} \in\left[\xi_{i}, \xi_{i+1}\right], i=1,2, \ldots,(b-a) / h_{n}$. It is now sufficient to choose for every $i$ the points $\left(x_{i}, m_{i}\right)$ and $\left(x_{i}, j h_{n}\right), j=1, \ldots,\left\lfloor m_{i} / h_{n}\right\rfloor$.

The cardinality of the resulting mesh of the form (9) is

$$
\begin{gathered}
\mathcal{N}_{C L}(n)=\sum_{i=1}^{(b-a) / h_{n}}\left(1+\left\lfloor m_{i} / h_{n}\right\rfloor\right)>\frac{1}{h_{n}^{2}} \sum_{i=1}^{(b-a) / h_{n}} g\left(x_{i}\right) h_{n} \\
\geq \frac{1}{\left(\delta_{n} / \sqrt{2}\right)^{2}} \sum_{i=1}^{(b-a) / h_{n}} g\left(x_{i}\right) h_{n} \sim \frac{M^{2} n^{4}}{2 c^{2}} \int_{a}^{b} g(x) d x, \quad n \rightarrow \infty,
\end{gathered}
$$

since the second sum is an upper Riemann sum for the continuous function $g$. We stress that the approximation given by the Riemann sum is indeed already sharp at small $n$, since the step $h_{n} \sim \delta_{n} / \sqrt{2}$ is small and naturally adapted to both, the shape of $g$ and the length of the integration interval, cf. (4)-(7).

In order to implement the geometric construction of Lemma 1, obtaining the same constant of the mesh above, $C=1 /(1-\lambda(c))$, it is sufficient to choose $\tilde{c}$ such that $1-\lambda(c)=(1-\lambda(\tilde{c})) \cos (\pi / 2 \mu)$, i.e., $\tilde{c}$ solves the equation

$$
4 \tilde{c} e^{2 \tilde{c}}=1-\frac{1-4 c e^{2 c}}{\cos (\pi / 2 \mu)}
$$

where $0<c<c^{*}$ and $\mu>\frac{\pi}{2 \arccos (1-\lambda(c))}$; cf. (6)-(10). The cardinality of the resulting mesh turns out to be

$$
\mathcal{N}_{G}(n)=\lceil\mu n\rceil \frac{b-a}{h_{n}(\tilde{c})} \sim\lceil\mu n\rceil\left\lceil\frac{(b-a) M n^{2}}{\sqrt{2} \tilde{c}}\right\rceil \sim \frac{\mu M(b-a)}{\sqrt{2} \tilde{c}} n^{3}, n \rightarrow \infty .
$$

We have implemented a Matlab code that computes such a sub-optimal polynomial mesh, by adaptive estimation of the Lipschitz constant and numerical computation of $g$ extrema; cf. [14].

Finally, we can estimate the ratio of the cardinalities as

$$
\frac{\mathcal{N}_{C L}(n)}{\mathcal{N}_{G}(n)} \gtrsim \frac{\sqrt{2} \tilde{c}}{2 \mu c^{2}} M \frac{\int_{a}^{b} g(x) d x}{b-a} n, \quad n \rightarrow \infty ;
$$

notice that the second factor is purely geometric and depends on the shape of the Lipschitz graph domain (through the product of the Markov constant $M$ with the integral mean of $g$ ), whereas the first one depends on the choice of the parameters $\mu$ and $c$. Choosing for instance $c$ such that the constant of the meshes is $C=2$ and $\mu=2$, we get $c \approx 0.1019, \tilde{c} \approx 0.0644$, and

$$
\frac{\mathcal{N}_{C L}(n)}{\mathcal{N}_{G}(n)} \gtrsim \alpha n, \quad n \rightarrow \infty, \quad \alpha=2.19 M \frac{\int_{a}^{b} g(x) d x}{b-a} .
$$

Inequality (13) actually shows that the construction of Lemma 1 is more adapted to the geometry of the graph domain $G$, and that the cardinality 
$\mathcal{N}_{G}(n)$ of sub-optimal meshes can be much smaller than $\mathcal{N}_{C L}(n)$ already at small values of the degree.

Consider for example the graph domain $G=\{(x, y): 0 \leq x \leq 1,0 \leq$ $y \leq g(x)=\exp (|x-0.5|)\}$, where we obtain $M \approx 13.18$ and $\alpha \approx 37.45$. For $n=8$ we have that the ratio of cardinalities is approximately 299.79 (and the estimate from below in (13) is approximately 299.63). Indeed, Lemma 1 gives a mesh made of about 148000 points (with approximately 9200 points on each of the 16 norming curves (11)), to be compared with the CL-mesh which has more than 44 millions points.

Observe that there is a simple way to reduce these cardinalities, at the price of increasing slightly the polynomial mesh constant. In fact, it is clear from the definition of polynomial mesh (1), that if one has a mesh for a compact set $K$, say $\mathcal{A}_{n}$ with constant $C_{1}$, and a mesh for a compact subset $H \subset K$, say $\mathcal{B}_{n}$ with constant $C_{2}$, then $\left(\mathcal{A}_{n} \cap(K \backslash H)\right) \cup \mathcal{B}_{n}$ is still a mesh for $K$ with constant $C_{1} C_{2}$.

For the graph domain $K=G$, one can choose for instance $H=[a, b] \times$ $[0, \min g]$, and $\mathcal{B}_{n}$ a $2 n \times 2 n$ Chebyshev grid (having mesh constant $C_{2}=2$, cf. [6]). In such a way, we can reduce the cardinality $\mathcal{N}_{G}$ of the example above from about 148000 to about 43000 , and $\mathcal{N}_{C L}$ from about 44 millions to about 4.3 millions (with a ratio approximately 100), both meshes having now a mesh constant $C=4$.

\section{References}

[1] M.S. Baouendi and C. Goulaouic, Approximation polynomiale de fonctions $C^{\infty}$ et analytiques, Ann. Inst. Fourier (Grenoble) 21 (1971), 139173.

[2] T. Bloom, L. Bos, J.-P. Calvi and N. Levenberg, Polynomial interpolation and approximation in $\mathbb{C}^{d}$, Ann. Polon. Math. 106 (2012), 53-81.

[3] L. Bos, J.-P. Calvi, N. Levenberg, A. Sommariva and M. Vianello, Geometric Weakly Admissible Meshes, Discrete Least Squares Approximation and Approximate Fekete Points, Math. Comp. 80 (2011), 16011621 .

[4] L. Bos, S. De Marchi, A. Sommariva and M. Vianello, Computing multivariate Fekete and Leja points by numerical linear algebra, SIAM J. Numer. Anal. 48 (2010), 1984-1999.

[5] L. Bos, A. Sommariva and M. Vianello, Least-squares polynomial approximation on weakly admissible meshes: disk and triangle, J. Comput. Appl. Math. 235 (2010), 660-668.

[6] L. Bos and M. Vianello, Low cardinality admissible meshes on quadrangles, triangles and disks, Math. Inequal. Appl. 15 (2012), 229-235. 
[7] J.P. Calvi and N. Levenberg, Uniform approximation by discrete least squares polynomials, J. Approx. Theory 152 (2008), 82-100.

[8] M.C. Delfour and J.-P. Zolésio, Shapes and Geometries, SIAM, Philadelphia, 2011.

[9] H. Ehlich and K. Zeller, Schwankung von Polynomen zwischen Gitterpunkten, Math. Z. 86 (1964), 41-44.

[10] M. Gentile, A. Sommariva and M. Vianello, Polynomial interpolation and cubature over polygons, J. Comput. Appl. Math. 235 (2011), 52325239 .

[11] A. Kroó, On optimal polynomial meshes, J. Approx. Theory 163 (2011), $1107-1124$.

[12] R. Pasquetti and F. Rapetti, Spectral element methods on unstructured meshes: which interpolation points?, Numer. Algorithms 55 (2010), 349-366.

[13] F. Piazzon and M. Vianello, Small perturbations of polynomial meshes, Appl. Anal. 92 (2013), 1063-1073.

[14] F. Piazzon and M. Vianello, lipgraphmesh: a Matlab function to compute polynomial meshes on planar Lipschitz graph domains, available online at http://www.math.unipd.it/ marcov/CAAsoft.

[15] W. Pleśniak, Nearly optimal meshes in subanalytic sets, Numer. Algorithms 60 (2012), 545-553.

[16] D. R. Wilhelmsen, A Markov inequality in several dimensions, J. Approx. Theory 11 (1974), 216-220.

[17] P. Zitnan, The collocation solution of Poisson problems based on approximate Fekete points, Eng. Anal. Bound. Elem. 35 (2011), 594-599. 